\title{
Syntheses of Potent, Selective and Orally Bioavailable Indazole-pyridine Series of Protein kinase B/Akt Inhibitors with Reduced Hypotension
}

Gui-Dong Zhu, ${ }^{1}$ Viraj B. Gandhi, ${ }^{1}$ Jianchun Gong, ${ }^{1}$ Sheela Thomas, ${ }^{1}$ Keith W. Woods, ${ }^{1}$ Xiaohong Song, ${ }^{1}$ Tongmei Li, ${ }^{1}$ R. Bruce Diebold, ${ }^{1}$ Yan Luo, ${ }^{1}$ Xuesong Liu, ${ }^{1}$ Ran Guan, ${ }^{1}$ Vered Klinghofer, ${ }^{1}$ Eric F. Johnson, ${ }^{1}$ Jennifer Bouska, ${ }^{1}$ Amanda Olson, ${ }^{1}$ Kennan C. Marsh, ${ }^{2}$ Vincent S. Stoll, ${ }^{3}$ Mulugeta Mamo, ${ }^{3}$ James Polakowski, ${ }^{4}$ Thomas J. Campbell, ${ }^{4}$ Thomas D. Penning, ${ }^{1}$ Qun Li, ${ }^{1}$ Saul H. Rosenberg, ${ }^{1}$ Vincent L. Giranda ${ }^{1}$

${ }^{1}$ Cancer Research, ${ }^{2}$ Preclinical Safety, ${ }^{3}$ Structural Biology, ${ }^{4}$ Integrative Pharmacology, GPRD, Abbott Laboratories

100 Abbott Park Road, Abbott Park, IL 60064-6101

\section{Supporting Information}

Analytical Data for Target Compounds

\begin{tabular}{|c|c|c|c|}
\hline & & \multicolumn{2}{|c|}{ Elemental Analysis } \\
\hline Compound & Formula & Calculated & Found \\
\hline $13 \mathrm{e}$ & $\mathrm{C}_{22} \mathrm{H}_{19} \mathrm{~F}_{3} \mathrm{~N}_{4} \mathrm{O}=2.8 \mathrm{TFA}$ & $\mathrm{C}, 45.31 ; \mathrm{H}, 3.00 ; \mathrm{N}, 7.66$ & $\mathrm{C}, 45.59 ; \mathrm{H}, 2.73 ; \mathrm{N}, 7.84$ \\
\hline $13 f$ & $\mathrm{C}_{22} \mathrm{H}_{19} \mathrm{~F}_{3} \mathrm{~N}_{4} \mathrm{O}=3.0$ TFA & $\mathrm{C}, 44.57 ; \mathrm{H}, 2.94 ; \mathrm{N}, 7.43$ & $\mathrm{C}, 44.47 ; \mathrm{H}, 2.78 ; \mathrm{N}, 7.46$ \\
\hline $13 \mathbf{i}$ & $\mathrm{C}_{22} \mathrm{H}_{21} \mathrm{ClN}_{4} \mathrm{O}=3$ TFA & $\mathrm{C}, 45.76 ; \mathrm{H}, 3.29 ; \mathrm{N}, 7.62$ & $\mathrm{C}, 45.86 ; \mathrm{H}, 3.28 ; \mathrm{N}, 7.72$ \\
\hline 13j & $\mathrm{C}_{22} \mathrm{H}_{21} \mathrm{BrN}_{4} \mathrm{O}=2.9$ TFA & $\mathrm{C}, 43.48 ; \mathrm{H}, 3.14 ; \mathrm{N}, 7.30$ & $\mathrm{C}, 43.67 ; \mathrm{H}, 3.06 ; \mathrm{N}, 7.10$ \\
\hline 13n & $\mathrm{C}_{22} \mathrm{H}_{20} \mathrm{ClFN}_{4} \mathrm{O}=2.7 \mathrm{TFA}$ & $\mathrm{C}, 45.79 ; \mathrm{H}, 3.18 ; \mathrm{N}, 7.80$ & $\mathrm{C}, 45.86 ; \mathrm{H}, 3.44 ; \mathrm{N}, 7.66$ \\
\hline 13o & $\mathrm{C}_{22} \mathrm{H}_{20} \mathrm{ClFN}_{4} \mathrm{O}-2.7 \mathrm{TFA}$ & $\mathrm{C}, 45.79 ; \mathrm{H}, 3.18 ; \mathrm{N}, 7.80$ & $\mathrm{C}, 45.86 ; \mathrm{H}, 3.44 ; \mathrm{N}, 7.66$ \\
\hline $13 p$ & $\mathrm{C}_{22} \mathrm{H}_{20} \mathrm{Cl}_{2} \mathrm{~N}_{4} \mathrm{O}=3.5 \mathrm{TFA}$ & $\mathrm{C}, 42.15 ; \mathrm{H}, 2.87 ; \mathrm{N}, 6.78$ & $\mathrm{C}, 41.86 ; \mathrm{H}, 3.05 ; \mathrm{N}, 6.60$ \\
\hline $13 r$ & $\mathrm{C}_{22} \mathrm{H}_{20} \mathrm{Cl}_{2} \mathrm{~N}_{4} \mathrm{O}=3.2 \mathrm{TFA}$ & $\mathrm{C}, 43.38 ; \mathrm{H}, 2.98 ; \mathrm{N}, 7.18$ & $\mathrm{C}, 43.49 ; \mathrm{H}, 3.18 ; \mathrm{N}, 6.92$ \\
\hline $13 \mathrm{~s}$ & $\mathrm{C}_{22} \mathrm{H}_{20} \mathrm{BrFN}_{4} \mathrm{O}=2.8 \mathrm{TFA}$ & $\mathrm{C}, 42.80 ; \mathrm{H}, 2.97 ; \mathrm{N}, 7.23$ & $\mathrm{C}, 42.73 ; \mathrm{H}, 3.17 ; \mathrm{N}, 7.02$ \\
\hline $13 \mathrm{t}$ & $\mathrm{C}_{22} \mathrm{H}_{20} \mathrm{BrFN}_{4} \mathrm{O}=2.6$ TFA & $\mathrm{C}, 43.46 ; \mathrm{H}, 3.03 ; \mathrm{N}, 7.45$ & $\mathrm{C}, 43.52 ; \mathrm{H}, 3.07 ; \mathrm{N}, 7.43$ \\
\hline $13 u$ & $\mathrm{C}_{22} \mathrm{H}_{19} \mathrm{BrF}_{2} \mathrm{~N}_{4} \mathrm{O}=3 \mathrm{TFA}$ & $\mathrm{C}, 41.24 ; \mathrm{H}, 2.72 ; \mathrm{N}, 6.87$ & $\mathrm{C}, 41.35 ; \mathrm{H}, 2.68 ; \mathrm{N}, 6.76$ \\
\hline$\overline{13 v}$ & $\mathrm{C}_{23} \mathrm{H}_{23} \mathrm{FN}_{4} \mathrm{O}=2.6$ TFA & $\mathrm{C}, 49.31 ; \mathrm{H}, 3.76 ; \mathrm{N}, 8.16$ & $\mathrm{C}, 49.28 ; \mathrm{H}, 3.61 ; \mathrm{N}, 8.10$ \\
\hline $13 w$ & $\mathrm{C}_{23} \mathrm{H}_{23} \mathrm{BrN}_{4} \mathrm{O} \cdot 2.7$ TFA & $\mathrm{C}, 44.93 ; \mathrm{H}, 3.41 ; \mathrm{N}, 7.38$ & $\mathrm{C}, 45.11 ; \mathrm{H}, 3.36 ; \mathrm{N}, 7.28$ \\
\hline $13 x$ & $\mathrm{C}_{23} \mathrm{H}_{23} \mathrm{FN}_{4} \mathrm{O}=3$ TFA & $\mathrm{C}, 50.79 ; \mathrm{H}, 3.91 ; \mathrm{N}, 8.58$ & $\mathrm{C}, 51.10 ; \mathrm{H}, 4.02 ; \mathrm{N}, 8.43$ \\
\hline $13 y$ & $\mathrm{C}_{23} \mathrm{H}_{23} \mathrm{FN}_{4} \mathrm{O}=2.8 \mathrm{TFA}$ & $\mathrm{C}, 48.40 ; \mathrm{H}, 3.66 ; \mathrm{N}, 7.89$ & $\mathrm{C}, 48.59 ; \mathrm{H}, 3.85 ; \mathrm{N}, 7.90$ \\
\hline $13 z$ & $\mathrm{C}_{23} \mathrm{H}_{23} \mathrm{FN}_{4} \mathrm{O}=2.9 \mathrm{TFA}$ & $\mathrm{C}, 47.97 ; \mathrm{H}, 3.62 ; \mathrm{N}, 7.77$ & $\mathrm{C}, 48.09 ; \mathrm{H}, 3.75 ; \mathrm{N}, 7.87$ \\
\hline 13aa & $\mathrm{C}_{25} \mathrm{H}_{28} \mathrm{~N}_{4} \mathrm{O}=2.5$ TFA & $\mathrm{C}, 52.56 ; \mathrm{H}, 4.48 ; \mathrm{N}, 8.17$ & $\mathrm{C}, 52.49 ; \mathrm{H}, 4.41 ; \mathrm{N}, 8.06$ \\
\hline $13 \mathbf{a b}$ & $\mathrm{C}_{23} \mathrm{H}_{23} \mathrm{FN}_{4} \mathrm{O}_{2}=3.5 \mathrm{TFA}$ & $\mathrm{C}, 44.73 ; \mathrm{H}, 3.32 ; \mathrm{N}, 6.96$ & $\mathrm{C}, 44.77 ; \mathrm{H}, 3.46 ; \mathrm{N}, 6.83$ \\
\hline 13ad & $\mathrm{C}_{23} \mathrm{H}_{21} \mathrm{~F}_{3} \mathrm{~N}_{4} \mathrm{O}-2.7$ TFA & $\mathrm{C}, 46.45 ; \mathrm{H}, 3.25 ; \mathrm{N}, 7.63$ & $\mathrm{C}, 46.55 ; \mathrm{H}, 3.30 ; \mathrm{N}, 7.64$ \\
\hline 13ae & $\mathrm{C}_{24} \mathrm{H}_{20} \mathrm{~F}_{6} \mathrm{~N}_{4} \mathrm{O}-2.8$ TFA & $\mathrm{C}, 43.69 ; \mathrm{H}, 2.82 ; \mathrm{N}, 6.89$ & $\mathrm{C}, 43.70 ; \mathrm{H}, 2.65 ; \mathrm{N}, 6.82$ \\
\hline 13ah & $\mathrm{C}_{23} \mathrm{H}_{20} \mathrm{~F}_{4} \mathrm{~N}_{4} \mathrm{O}-2.7$ TFA & $\mathrm{C}, 45.34 ; \mathrm{H}, 3.04 ; \mathrm{N}, 7.45$ & $\mathrm{C}, 45.51 ; \mathrm{H}, 2.90 ; \mathrm{N}, 7.40$ \\
\hline 13al & $\mathrm{C}_{23} \mathrm{H}_{21} \mathrm{~F}_{3} \mathrm{~N}_{4} \mathrm{O}_{2}-2.6$ TFA & $\mathrm{C}, 45.84 ; \mathrm{H}, 3.22 ; \mathrm{N}, 7.58$ & $\mathrm{C}, 45.87 ; \mathrm{H}, 3.17 ; \mathrm{N}, 7.28$ \\
\hline 13am & $\mathrm{C}_{23} \mathrm{H}_{21} \mathrm{~F}_{3} \mathrm{~N}_{4} \mathrm{O}_{2} \cdot 2.4$ TFA & $\mathrm{C}, 46.63 ; \mathrm{H}, 3.29 ; \mathrm{N}, 7.82$ & $\mathrm{C}, 46.57 ; \mathrm{H}, 3.30 ; \mathrm{N}, 7.80$ \\
\hline 13an & $\mathrm{C}_{28} \mathrm{H}_{26} \mathrm{~N}_{4} \mathrm{O}=3.2 \mathrm{TFA}$ & $\mathrm{C}, 51.68 ; \mathrm{H}, 3.68 ; \mathrm{N}, 7.01$ & $\mathrm{C}, 51.94 ; \mathrm{H}, 3.66 ; \mathrm{N}, 6.85$ \\
\hline $13 \mathbf{a o}$ & $\mathrm{C}_{23} \mathrm{H}_{22} \mathrm{~N}_{4} \mathrm{O}_{3}-2.6$ TFA & $\mathrm{C}, 48.46 ; \mathrm{H}, 3.55 ; \mathrm{N}, 8.02$ & $\mathrm{C}, 48.59 ; \mathrm{H}, 3.65 ; \mathrm{N}, 8.13$ \\
\hline 13ap & $\mathrm{C}_{23} \mathrm{H}_{20} \mathrm{~F}_{2} \mathrm{~N}_{4} \mathrm{O}_{3} \bullet 3$ TFA & $\mathrm{C}, 44.63 ; \mathrm{H}, 2.97 ; \mathrm{N}, 7.18$ & $\mathrm{C}, 44.74 ; \mathrm{H}, 3.08 ; \mathrm{N}, 7.23$ \\
\hline 13aq & $\mathrm{C}_{23} \mathrm{H}_{20} \mathrm{~F}_{2} \mathrm{~N}_{4} \mathrm{O}_{3} \bullet 3.1$ TFA & $\mathrm{C}, 43.08 ; \mathrm{H}, 2.77 ; \mathrm{N}, 6.65$ & $\mathrm{C}, 42.94 ; \mathrm{H}, 2.79 ; \mathrm{N}, 6.64$ \\
\hline 13ar & $\mathrm{C}_{28} \mathrm{H}_{33} \mathrm{~N}_{5} \mathrm{O}_{3}=3.5$ TFA & $\mathrm{C}, 47.41 ; \mathrm{H}, 4.15 ; \mathrm{N}, 7.90$ & $\mathrm{C}, 47.32 ; \mathrm{H}, 4.05 ; \mathrm{N}, 7.80$ \\
\hline 13as & $\mathrm{C}_{29} \mathrm{H}_{35} \mathrm{~N}_{5} \mathrm{O}_{2}=3.3$ TFA & $\mathrm{C}, 49.61 ; \mathrm{H}, 4.48 ; \mathrm{N}, 8.13$ & $\mathrm{C}, 49.94 ; \mathrm{H}, 4.08 ; \mathrm{N}, 8.15$ \\
\hline 13au & $\mathrm{C}_{29} \mathrm{H}_{34} \mathrm{~N}_{4} \mathrm{O}_{2} \cdot 2.0$ TFA & $\mathrm{C}, 56.73 ; \mathrm{H}, 5.19 ; \mathrm{N}, 8.02$ & $\mathrm{C}, 56.59 ; \mathrm{H}, 5.08 ; \mathrm{N}, 7.90$ \\
\hline 13av & $\mathrm{C}_{29} \mathrm{H}_{35} \mathrm{~N}_{5} \mathrm{O}_{2} \cdot 3.6$ TFA & $\mathrm{C}, 48.52 ; \mathrm{H}, 4.34 ; \mathrm{N}, 7.82$ & $\mathrm{C}, 48.66 ; \mathrm{H}, 4.15 ; \mathrm{N}, 7.91$ \\
\hline
\end{tabular}




\begin{tabular}{|c|c|c|c|}
\hline 13aw & $\mathrm{C}_{24} \mathrm{H}_{27} \mathrm{~N}_{5} \mathrm{O}_{2} \bullet 3.5$ TFA & $\mathrm{C}, 45.60 ; \mathrm{H}, 3.76 ; \mathrm{N}, 8.58$ & $\mathrm{C}, 45.75 ; \mathrm{H}, 3.06 ; \mathrm{N}, 8.50$ \\
\hline $\mathbf{3 4}$ & $\mathrm{C}_{22} \mathrm{H}_{21} \mathrm{~N}_{3} \mathrm{O}_{2} \cdot 2.3$ TFA & $\mathrm{C}, 51.39 ; \mathrm{H}, 3.78 ; \mathrm{N}, 6.76$ & $\mathrm{C}, 51.48 ; \mathrm{H}, 3.63 ; \mathrm{N}, 6.75$ \\
\hline $\mathbf{3 5}$ & $\mathrm{C}_{27} \mathrm{H}_{24} \mathrm{~N}_{4} \mathrm{O}_{3} \cdot 2.8 \mathrm{HCl}$ & $\mathrm{C}, 60.21 ; \mathrm{H}, 5.02 ; \mathrm{N}, 10.40$ & $\mathrm{C}, 60.06 ; \mathrm{H}, 4.65 ; \mathrm{N}, 10.03$ \\
\hline 36 & $\mathrm{C}_{27} \mathrm{H}_{23} \mathrm{~N}_{3} \mathrm{O}_{3} \cdot 2.1 \mathrm{HCl}$ & $\mathrm{C}, 63.08 ; \mathrm{H}, 4.92 ; \mathrm{N}, 8.17$ & $\mathrm{C}, 62.89 ; \mathrm{H}, 5.02 ; \mathrm{N}, 7.93$ \\
\hline 37c & $\mathrm{C}_{22} \mathrm{H}_{20} \mathrm{~F}_{3} \mathrm{~N}_{5} \mathrm{O} \cdot 2.2$ TFA & $\mathrm{C}, 46.75 ; \mathrm{H}, 3.30 ; \mathrm{N}, 10.33$ & $\mathrm{C}, 46.99 ; \mathrm{H}, 2.91 ; \mathrm{N}, 10.29$ \\
\hline 37d & $\mathrm{C}_{21} \mathrm{H}_{19} \mathrm{~F}_{2} \mathrm{~N}_{5} \mathrm{O} \cdot 3.0$ TFA & $\mathrm{C}, 43.97 ; \mathrm{H}, 3.01 ; \mathrm{N}, 9.50$ & $\mathrm{C}, 44.19 ; \mathrm{H}, 3.02 ; \mathrm{N}, 9.51$ \\
\hline
\end{tabular}

*To whom correspondence should be addressed. Phone: 847-935-1305. FAX: 847-9355165. E-mail: e-mail: gui-dong.zhu@ abbott.com 